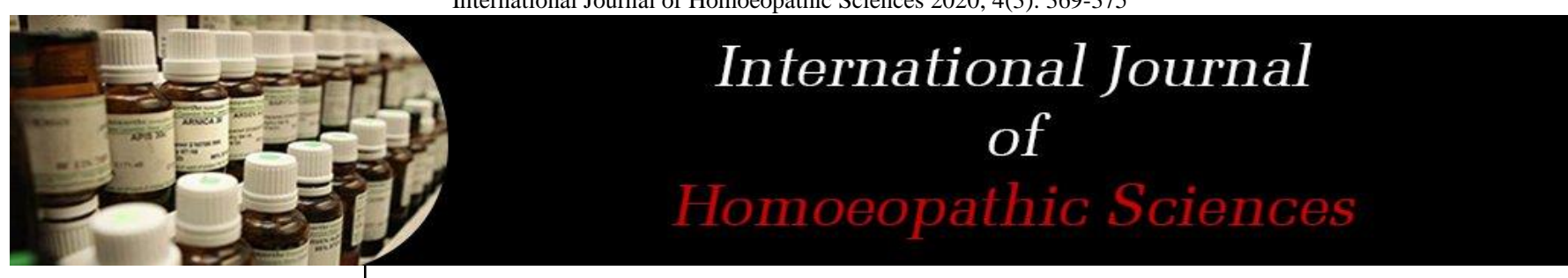

E-ISSN: 2616-4493 P-ISSN: 2616-4485 www.homoeopathicjournal.com IJHS 2020; 4(3): 369-375 Received: 22-06-2020 Accepted: 26-07-2020

Dr. Chakravarthy GS Srigiri Associate Professor, Department of Practice of Medicine, National Homoeopathy Research Institute in Mental Health, Kottayam, Kerala, India

Dr. KC Muraleedharan Officer In-Charge, Professor and HoD, Department of Practice of Medicine, National Homoeopathy Research Institute in Mental Health, Kottayam, Kerala, India

Corresponding Author: Dr. Chakravarthy GS Srigiri, Associate Professor, Department of Practice of Medicine, National Homoeopathy Research Institute in Mental Health, Kottayam, Kerala, India

\section{A review on coagulopathy in Covid-19 and its Homoeopathic management}

\author{
Dr. Chakravarthy GS Srigiri and Dr. KC Muraleedharan
}

DOI: https://doi.org/10.33545/26164485.2020.v4.i3f.239

\begin{abstract}
Background: Corona Virus disease (Covid-19) rapidly spreading pandemic was initially found as a cluster of severe pneumonia cases in December 2019 reported in Wuhan, Hubei province, China. Infection with the virus, can be asymptomatic or mild to severe symptomatic disease causing Acute viral pneumonia like symptoms leading to fatality in the least possible time if untreated. The pathological cause of this is severe coagulopathies in the microvasculature primarily in the lungs causing hypoxia and later multiorgan failure. Though thrombolytics play an important role, it fail to recover the patient completely, and if so may have thrombolytic effects in other organs too which lead to complications. Selection of Homoeopathic similimum may be practically difficult in such acute condition in an ICU setup but Homoeopathy Remedies which act primarily on thrombosis phenomenon along with affinity on pulmonary organs need to be selected and administered as an adjuvant therapy for beneficial results. Primarily the Tissue remedies like Kali mur, Natsulph, Ferrum phos, and remedies like arnica, Arsenic album, Ammonium Carb, animal Remedies from Ophidia group are found to be effective equally in thrombolysis and prevents further complications.

Objectives: The primary focus of this article is on causation of coagulation mechanism in Covid-19 disease, resulting in multiorgan failure and scope of Homoeopathic Medicines as Adjuvant therapy in preventing thromboembolic complications.

Methods: Method: Information was obtained from the latest articles on coagulation mechanism in covid-19 research articles and importance of Homoepathic medicines in preventing coagulation. Homoeopathic remedies were chosen from the latest Articles, various original literature and Repertories and their physiological pharmacological and Homoeopathic action on prevention of the formation of thrombus and their role in effective thrombolysis is explained.

Conclusion: The COVID-19-associated coagulopathy (CAC) are distinct from those seen with bacterial Sepsis-Induced Coagulopathy (SIC) and disseminated intravascular coagulation (DIC). The CAC usually shows increased D-dimer and fibrinogen levels with minimal abnormalities in Prothrombin time and platelet count. Homoeopathic remedies are proved to be effective in thrombolysis can be thought of primarily as an adjuvant to conventional treatment for an early recovery.
\end{abstract}

Keywords: Coagulation mechanism, Covid-19 coagulopathy, homoeopathic tissue remedies, ammonium carb, arsenic album, Lachesis, Bothrops, Crotalus, Vipera

\section{Introduction}

Corona virus disease 2019 (COVID-19) caused by severe acute respiratory syndrome coronavirus 2 (SARS-COV-2) is an ongoing global health emergency. The disease name was subsequently recommended as COVID-19 by the World Health Organization. Meanwhile 2019-ncov was renamed SARS-CoV-2 by the International Committee on Taxonomy of Viruses. The transmission of this virus is via droplets and close contact and Feaco-oral transmission

The median duration of RNA detection in the stool was 22 days in the sputum and saliva28days and in the serum 16 days. SARS-Cov-2 binds to host endothelial cells via the Angiotensin converting enzyme (ACE-2) receptor(R)-a metallo peptidase which is present in all major organs particularly high in the lungs, heart, veins and arteries. Its protein Expression causes macro and micro vascular thrombosis are formed through the gap junctions between pericytes of blood vessels in all major organs ${ }^{[2]}$.

Platelets after getting exposed to Covid infection triggers the release of Platelet microbial proteins, peptides, Platelet factor (PF)-4, RANTES, and fibrino peptide B. The induction of a cytokine storm, suppression of antiviral immunity and the activation of proinflammatory response is the root cause of pathogenecity both in SARS and COVID-19. CoV-2 differs from SARS-CoV in interferon-antagonizing and inflammasome-activating properties ${ }^{[3]}$. 


\section{Clotting Mechanism ${ }^{[4]}$.}

After injury to endothelium, there is activation of pericytes and fibroblasts. Simultaneously Activated factor VIIa circulating in blood binds with tissue factor (TF) and activates the factor $\mathrm{X}$ to $\mathrm{Xa}$ generating thrombin. This thrombin activates platelets via Protease activated receptors. The platelets adhere to Subendothelial collagen via Glycoprotein IB (GP IB) receptors. Gp IB receptors in turn bind to factor XI which helps in localising factor VIII to site of endothelial disruption via carrier protein VWF. Factor XIa mediates activation of factor IX to IXa, wherein Factor VIIIa serves as cofactor in this mechanism. Factor IXa mediates activation of $\mathrm{X}$ to $\mathrm{Xa}$ where in Factor Va serves as a cofactor for this mechanism. Meanwhile partially activated Factor $\mathrm{V}$ is released from Platelet Granules upon platelet activation. Factors V, VIII, XI are involved in sustained procoagulant responses (Intrinsic pathway).

Single thrombin activated platelet exposes 12000 copies of receptors that concentrate fibrinogen for fibrin formation. The Fibrinogen with factor XIII is the final thrombin substrates that stabilize Haemostatic plug.

To avoid interruption in microvasculature of various organs an inhibitory mechanism for coagulation is regulated by factors like - i) TF pathway inhibitor which Neutralises factor Xa when in complex with TF-FVIIa and ii) Antithrombin Neutralises Xa \& thrombin. These inhibitors are increased only when TF is exposed at high level ${ }^{[4]}$.

\section{Fibrin and D-Dimers ${ }^{[5,6]}$.}

During clotting mechanism plasma fibrinogen is converted into fibrin monomers by enzymatic thrombin. These Monomers are linked at $\mathrm{C}$ terminal appendages of the $\gamma$ chains by factor XIIIa resulting in dimerisations and form the clot. Later as a part of thrombolysis by plasmin, proteolysis of this "crosslinked" fibrin generates fragments D-Dimers and E as terminal products. If proteolysis occurs in direct fibrinogen or non-cross linked fibrin, (non-XL-Fg) it results in formation of monomer fragment $\mathrm{D}$. Hence the dimeric D-domain therefore may serve as indicator of in vivo fibrin formation.

The values of D-Dimers are an index of fibrin turnover in the circulation and a single measurement is adequate to assess the fibrinolytic status. As D-Dimers are not artificially generated in vitro during blood collection, its measurement more consistently reflects in vivo haemostatic activity. Its absence excludes the presence of intravascular thrombus.

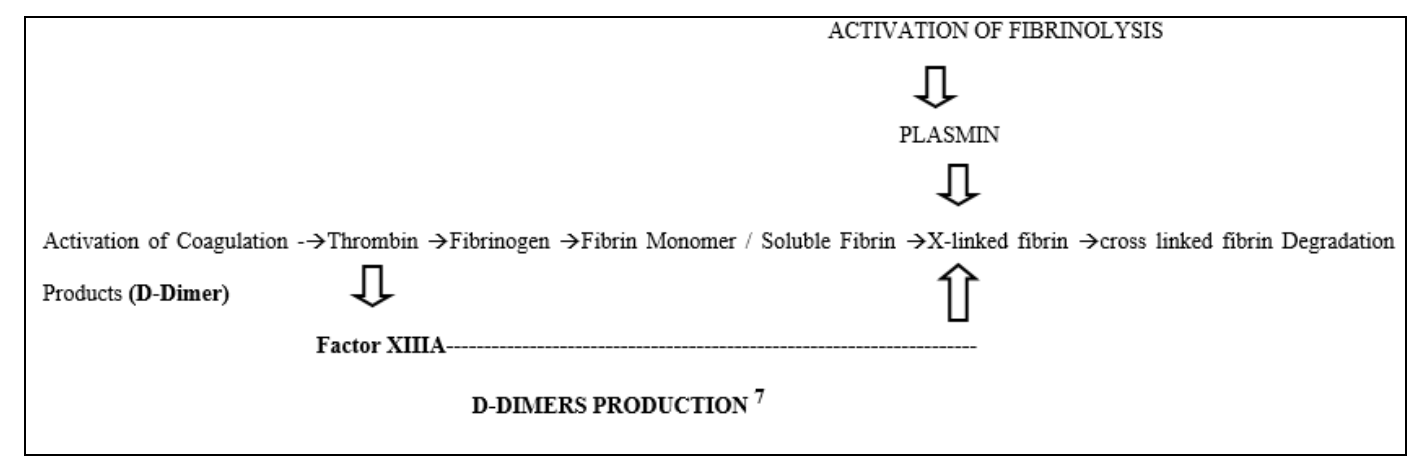

Coagulopathy activation in covid-19: Pulmonary vascular thrombosis may occur as a result of hypoxia, which stimulates coagulation. Within hours of infection with Covid infection proinflammatory reactions takes place resulting in anaphylatoxins (c3a and c5a) release, and C5a signaling protein activates cytokine storm. A hypercoagulable inflammatory state in small vessels of lungs causes diffuse alveolar damage. Later lymphocyte exhaustion, immune paresis, Imbalances between complement, extrinsic and intrinsic coagulation pathways contributes to a net procoagulant state in the microvasculature of various critical organs leading to multi organ damage ${ }^{[8]}$. Most cases of COVID -19 lung infections showed signs of pale areas with firm, reddish-blue areas with high capillary-to-fiber ratios ${ }^{[9]}$.

In many cases laboratory tests showed elevated levels of Ddimer, Raised C-reactive protein, increased Lactate Dehydrogenase (indicating tissue Damage) as well as mild thrombocytopenia. But abnormalities in prothrombin time, partial thromboplastin time, and platelet counts are relatively uncommon in initial presentations. The NLRP3* inflammasome is a critical component of the innate immune system that mediates caspase- 1 activation and the secretion of proinflammatory cytokines (IL-1 $\beta / \mathrm{IL}-18$ ) in response to microbial infection and cellular damage play a key role in coagulopathy. Elevated cardiac enzyme concentrations with normal fibrinogen and platelet levels are early features of severe pulmonary intravascular coagulopathy $[9,10,11]$.

Elevated D-dimer at admission is associated with increased mortality. Rising D-dimer after admission precedes multiorgan failure \& Longer duration of hospital stay associated with increasing D-dimer indicates development of Sepsis physiology.

Investigations and assesment of coagulopathy ${ }^{[12]}$. Coagulopathy defined as spontaneous prolongation of the prothrombin time $>3$ seconds or activated partial thromboplastin time $>5$ seconds as independent predictors of thrombotic complications.

Coagulopathy reflects resultant thrombo inflammation and not intrinsic viral activity.

\section{Evaluation of Coagulopathy in COVID-19 case $^{[13]}$.}

The routine investigations for Covid-19 coagulopathies to be followed are:

- Hemostasis function, Lymphocyte count

- Significantly elevated D-dimer level.

- Prolonged Prothrombin time (PT)

- Platelet count

- Fibrinogen 
Table 1: The Isth Score (International Society on Thrombosis and Haemostasis)

\begin{tabular}{|c|c|c|}
\hline Platelet count & $\begin{array}{c}2 \text { points if }<50 \times 10^{9} / \mathrm{L} \\
1 \text { point if } \geq 50 \times 10^{9} / \mathrm{L} \text { but }<100 \times 10^{9} / \mathrm{L} \\
0 \text { points if }>100 \times 10^{9} / \mathrm{L}\end{array}$ & $\begin{array}{c}2 \text { points if }<100 \times 10^{9} / \mathrm{L} \\
1 \text { point if } 100 \times 10^{9} / \mathrm{L} \text { but }<150 \times 10^{9} / \mathrm{L}\end{array}$ \\
\hline $\begin{array}{c}\text { FDP or } \\
\text { D-dimer level }\end{array}$ & $\begin{array}{l}3 \text { points if increased in four fold } \\
2 \text { points if increase } 2 \text { to } 4 \text { fold } \\
0 \text { points if increase }<\text { two fold }\end{array}$ & NA \\
\hline PT & $\begin{array}{c}2 \text { points if } \geq 6 \text { seconds } \\
1 \text { point if } \geq 3 \text { seconds but }<6 \text { seconds } \\
0 \text { points if }<3 \text { seconds }\end{array}$ & $\begin{array}{l}2 \text { points if } \mathrm{PT} \text { ratio }>1.4 \\
1 \text { point if } \mathrm{PT} \text { ratio }>1.2 \text { but } \leq 1.4\end{array}$ \\
\hline Fibrinogen & $\begin{array}{l}1 \text { point for }<1 \mathrm{~g} / \mathrm{L} \\
0 \text { points for }>1 \mathrm{~g} / \mathrm{L}\end{array}$ & NA \\
\hline SOFA score* & NA & $\begin{array}{l}2 \text { points if score } \geq 2 \\
1 \text { point if score } 1\end{array}$ \\
\hline Positive score & Score $\geq 5$ suggests overt DIC & Score $\geq 4$ suggests of SIC \\
\hline
\end{tabular}

DIC - disseminated Intravascular coagulation; FDP, fibrin degradation product; PT - Prothrombin time; SIC, Sepsis-induced coagulopathy; SOFA - Sequential Organ Failure Assessment. NA, not applicable;

Patients for DIC are evaluated by ISTH DIC score; if score of $<5$, DIC is unlikely and score should be recalculated every $1-2$ days as required.

\section{Special Investigations:}

- CT SCAN LUNGS

- A Ventilation / Perfusion scan (V/Q) lung scan followed by pulmonary Angiography in conjunction with a low probability lung scan is generally considered to be the standard diagnostic strategy.

- DD when measured with a quantitative assay, is nearly always increased in acute PE (sensitivity 85\%-99\%), and a low value virtually rules it out.

\section{Management}

Coagulopathy profile should be monitored frequently as depending on the severity of the case. It should be managed as it would be for any critically ill patient, following the established practice of using thromboembolic prophylaxis for critically ill hospitalized patients, and standard supportive care measures for those with sepsis-induced coagulopathy or DIC.

Presently the current data do not suggest the use of full intensity anticoagulation doses unless otherwise clinically indicated ${ }^{[14]}$.

A metaanalysis reported decreased mortality with the use of early low-molecular weight heparin (LMWH) in a nonCOVID-19 ARDS population. It suggests that heparin may possess anti-viral properties by acting on SARS-CoV-2 surface receptor binding proteins and inhibiting viral attachment. ${ }^{15}$

\section{Homoeopathic Management of Thrombosis}

As the Important cause of Covid-19 deaths is Coagulopathies, focus is to be viewed on reducing complementary cascade activation \& Proinflammatory markers in the body preventing multi organ damage.

Homoeopathy the alternative system of medicine acts in the best way when prescribed according to the routine case taking, analysis evaluation and systematic Repertorization technique. But in acute severe Infectious diseases the method of systematic analysis and Repertorization may be overlooked due to the emergency care for the patient as the need of the hour.

Homoeopathy treatment is based on expressions of the symptoms individualistically in different manners, when the common manifestations of Covid-19 are taken out Repertorised, remedies like Arsenic alb, Bryonia alb, camphor, pulsatilla, etc are represented in the outcome result. But in a case of severe coagulopathy in ICU settings, remedies acting on reducing complementary cascade, inflammatory markers are required, though little information or clinical trials are available on this regards.

In this view some of the Homeopathic remedies which are having action on Thrombosis phenomenon were searched out. There are many Homoeopathic Remedies acting on blood vessels or plasma causing or initiating coagulation cascade. As an Alternative system of Medicine to give relief to the covid -19 sufferers, our Homoeopathic medicines can act as adjuvants where there are many failures in conventional system and still are under trials. Though many research trials are conducted showing Homoeopathic Medicines action at Nano-particulate level, and though not fully evidenced, these medicines can be used as adjuvant to Modern system of Medicine to save the suffering humanity from such deadly Epidemic.

\section{Role of Homoeopathic medicines as adjuvants ${ }^{[16]}$.}

- As non-specific potentiators of immune response by enhancing the immunogenicity of molecules without altering chemical composition.

- Increases efficiency of macrophage processing of antigen and retards the absorption of antigen and prolongation of exposure to antibody forming tissues.

- Enhance lymphokine activity.

Homeopathic remedies which cause thromboembolism and acting on circulation disturbances, taken from Murphy repertory, especially first and second grade remedies, with their indications and action at physiological level are mentioned below. The Remedies are taken from Murphy Repertory especially first and second grade Remedies ${ }^{[21]}$.

\section{Blood}

- Disorganization of Blood: Echinecea, Pyrog, Crot, Baptisia, Arsenic, Muriatic Acid

- Septicemia: Crot, Carboveg, Echi, Lach, Pyrog

- Regulates Watery Elements of Venous SystemNatsulph

- Thrombosis - Heart clot suspected in Pneumonia Ammonium carb

- RBC Decaying Rapidly - Kaliphos

- Plasma coloring matter escapes into blood - Arg.n

- Agranulocytosis - Lachesis 
- Coagulation formation of clots- causes plugs - kali mur

- Easy clottage to gelatinous mass - Merc.sol

- Sycotic pneumonia in - Nat Sulph

- Ailments from Septicemia / Blood poisoning - Lach

\section{Tissue Remedies}

In Homoeopathy Tissue remedies which were being neglected and does not see much role in routine practice. But William.H.Schuessler has invented this wonderful Biochemic system of medicine where this tissue salts supplements \& nullifies the imbalance of the salts in the tissue fluids. These tissue salts prepared in accordance with Homeopathic practice restores the fitness of these vital cells reduced practically of molecular form. The Micro doses are readily assimilated and absorbed immediately to nourish tiny cells of living organism.

Some of the few tissue remedies which has got tremendous action on thromboembolism as well as on Respiratory affections are Kalimur, Ferr.phos and Nat.sulph which can be thought of in this Covid-19 complication.

Kali Mur: Potassium is highly reactive and does not exist freely in nature. Reacts rapidly with water and decomposes it. It has Hygroscopic and hydrogenoid properties. Acts as most powerful reducing agent and Reacts with tissues or secretions having excess of $\mathrm{Co} 2, \mathrm{O} 2$, Hydrogen, Sulphur. Its deficiency produces acidosis in secretions and also indicated in hyperventilation and hypoxia. The molecular motion of potassium chloride in equilibrium maintains clot formation and its lysis. The lytic activity modifies the coagulation process preventive excess or continued clotting by normal molecular motion of this salt. At Physiologic level it causes Apoplexy, Thrombo embolic phenomenon, Swelling of lymphatic glands in infectious diseases, Pneumonia, Pleurisy, fibrinous exudations in lungs, Pneumonia ${ }^{[21]}$.

The Toxicological effects of Potassium chloride powder causes respiratory tract irritation and delayed pulmonary edema when inhaled, and gastrointestinal discomfort with nausea, vomiting and diarrhea when ingested ${ }^{[22]}$.

Homoeopathic Kalimur destroys the body wastes, and must be given during the convalescence and rebuilding of the health. It retards the further secretion mechanisms of the body and builds nitrogenous protein fibre fibrin. It is the most useful when inflammation starts receding and the membranes are thickening ${ }^{[28]}$.

The fibrin is the distinguished from albumen and its casien by seperation. The normal amount of fibrin is not held in proper solution without KCL. It causes fibrinous exudation (chemically related to fibrin) and dissolves grayish white secretions of mucus membranes and plastic exudations ${ }^{[21]}$. In case of deficiency of Kalimur the fibrin is non functional and is set free, discharged via nose and throat. If this kalimur is given fibrin becomes united with it and it restores the condition to normalcy. According to William Dewey" Clinical experience kalimur alternating with Ferrum phos is succesful in Second and third stages of pneumonia with white Viscid Expectoration and is also one of the best remedies for Asthma, Diphtheria with fibrinous exudations [26]. It is indicated in pneumonia during the stage of Hepatization with tough thick white sputum ${ }^{[22]}$. Dr.Carey recommends that a gargle of kalimur 10-15grains in glass of water should be used to remove the plastic exudations.

The only remedy given for Blood thick clotting under circulatory symptoms is Kali.mur. In conditions calling for
Kalimur the blood thickens to form clots ${ }^{[28]}$.

Ferrum Phos: Normally Iron phosphate helps in oxygenation to all parts of body. It Gives strength and toughness of circular walls of blood vessels and arteries and the muscular cells become flaccid through loss of iron, The Ferrum restores the tension, prevents Haemorrhages, and reduces hyperemia and fever ceases ${ }^{[24]}$.

This remedy is indicated during the first stages of catarrhal and febrile disturbances and inflammations before exudation has taken place. It is useful in acute bronchitis, capillary bronchitis, Broncho - Pneumonia, Acute Tuberculosis, Hemoptysis, bright Haemorrhage who suffers from bronchial catarrh, hyperemia of the lungs and the sputum is set free.

Ferrum Phos is useless when cyanosis has once taken place [22].

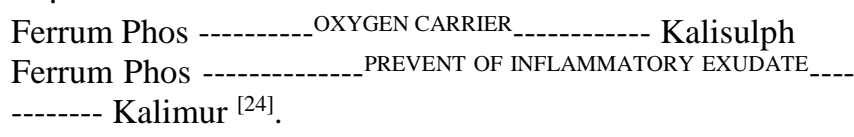

Natrum Sulph: Physiological Action.-This agent in small doses is a laxative, aperient and diuretic. It excites the secretions of the intestines and stimulates the activity of the intestinal ${ }^{[22]}$.

Found in intracellular fluid and principle action is to regulate water in tissue fluids. The organic waste products like lactic acid reacts with Natrum phos leaving a residue of water. Normally One molecule of Natrum sulph has capacity to carry two molecules of water and thus regulates excess of intra cellular fluids and eliminates excess of water from the blood. If excess water is present in blood it provides suitable cause of Plasmodium species breeding. This Natrum sulph help in Preventing the Hydrogenoid constitutions from Malarial Infections too. Some of the Important indications of Natrum sulph are Dyspnea during damp weather, Humid asthma, cough with thick greenish ropy expectoration, Delayed resolution in pneumonia, Asthma, Pain through left lower chest. ${ }^{24}$ Natrum sulph is the principle remedy in treatment of Influenza, Humid asthma and malaria.

Kali Carbonicum: This agent acts upon the mucous membranes of the respiratory and digestive tract, producing congestion. It is a diuretic and renders the urine alkaline. The serous membranes are dry and there are sticking pains. The fibrin of the blood is increased. It is beneficial in pulmonary affections the lower portion of the right lung with expectoration that is difficult and tenacious ${ }^{[22]}$.

\section{Ophidia Group}

Bothrops Lanceolatus: Description.-The Lance snake is of the family Crotolidae; found in the island of Martinique. Preparations.-The poison from this snake is triturated, and dilutions are made from the $6 x$ trituration ${ }^{[22]}$.

Bothrops is marked under remedies for Aphasia and also stroke Rubrics. But how does it compete with arnica in coagulopathies is to be differentiated. The differential with Arnica is an important one for several reasons, including the fact that because we know Arnica better, we give it more frequently. When the etiology (Injury) is from the outside, the remedy is more likely to be Arnica. When the etiology is an internal process the remedy is more likely to be Bothrops. At times Bothrops is indicated even in 
injuries where thromboembolus arises after effects of injuries. Though Bothrops is given under many repertories under bleeding conditions, but Bothrops is indicated especially in bleeding after intravascular clotting and thromboembolic phenomenon. ${ }^{\mathbf{1 6}}$

According to a study conducted, Bothrops has got both coagulant (clotted human fibrinogen indicating presence of Thrombin like enzyme) and anticoagulant properties (Increase in Partial thromboplastin time) ${ }^{[17]}$. Clinical experience by Dr.paul herscu says that Bothrops initiates. Thrombi Formation leads to Fullness Restlessness, Inflammation which is followed by Bleeding, Weakness and Paralysis which brings back to the segment of thrombus Formation. The bleeding in Bothrops is always secondary to the coagulation, so even though there is bleeding mentioned in many locations, it rarely shows up in practice. The thrombi are much more to the point. ${ }^{21}$

Lachesis: Preparations. - Triturations of the venom extracted from the live snake. Dilutions from the $6 x$ trituration ${ }^{[22]}$.

It Acts especially upon the blood, cerebro-spinal and ganglionic nervous systems, Pneumogastric nerve, Orifices and generative organs of woman. Often used in malignant diseases, (Malignant fevers, gangrene, malignant pustule), pyemia, phlebitis, putrid sore throat ${ }^{[23]}$.

It Produces irritation of the larynx, trachea, and the blood is decomposed leading to Haemorrhages causing low asthenic condition followed by unconsciousness. It is indicated in pneumonia when a septic condition is threatened. The expectoration consists of pus and blood, septic inflammations, offensiveness of the discharges ${ }^{[22]}$.

Vipera: Preparations: Triturations of the virus of this reptile and dilutions. Dosage: Trituration, 6x, and higher in dilutions. This remedy is indicated in phlebitis and varicose veins ${ }^{[22]}$

It acts physiologically on Human plasma, the venom of Vipera has both an immediate procoagulant effect and a late fibrinogenolytic and fibrinolytic activity. This procoagulant effect is shown in presence of calcium ions and is observed even in the absence of phospholipids in presence of Factors $\mathrm{V}$ and $\mathrm{X}$. The venom progressively hydrolyses fibrinogen impairing clot formation. It has a fibrinolytic action in the absence of Plasminogen.

On platelets, the venom of Vipera inhibits the segregation induced by ADP, collagen or small amounts of adrenalin. Platelets lose part of their procoagulant properties, platelet phospholipids and are hydrolysed causing hemolysis. On thromboelastogram it is evidenced that the clot formation of PRP is impaired ${ }^{[20]}$.

Arsenic ALB: Physiological Action - This agent when applied to the skin acts as a caustic, and produces a violent inflammation with sloughing. Small doses has a tonic effect upon the nervous system and circulation, Gastric and Intestinal secretions. Toxic doses produce violent gastroenteritis with nausea, diarrhea, Fatty degenerations of myocardium ensues. The red blood corpuscles are decreased in number, and the blood rendered less coaguable ${ }^{[22]}$.

The poison acts directly on the red corpuscles, diminishing their power of taking. up the oxygen supplied to them in the lungs; and the carbonaceous compounds thus unconsumed, deposit themselves in the form of fat., blood is black and noncoagulable.

Arsenic album as one of the constituents in a formulation has been shown to affect HT29 cells and human macrophages. Also, it showed $\downarrow N F-\kappa B$ hyperactivity (reduced expression of reporter gene GFP in transfect HT29 cells), $\downarrow$ TNF- $\alpha$ release in macrophages ${ }^{[25]}$.

Arnica Montana: Description: Natural Order, Compositae. Common Name:leopard's bane. Habitat, Northern Hemisphere. Preparations: The mother tincture is prepared from the fresh foot, root-leaves and full blown flower. The tincture for external use is prepared from the dry root and Dilutions from the mother tincture ${ }^{[22]}$.

A study conducted on Arnica in inflammatory process, there is no selective modulation of leukocyte subsets migration but only vascular regulations, regarding lymphatic absorption, CD54 expression, and histamine degranulation [18].

By administering Homoeopathic remedies to the sick, we aid them to help themselves and cure using the organisms natural available resources by modifying natural homeostatic mechanism. Arnica affects particularly the capillaries of secretions, hence useful in Haemorrhages and effusions, extravasations of blood and oedema after injuries [23].

A study on efficacy of Arnica 30 and 200 to thrombolyse a blood clot in an in vitro sample failed to prove but it may act in human organism once it comes in contact with endothelium and must be experimented further ${ }^{[19]}$.

Ammonium CARB: Physiological Action: This agent is a stimulant, diaphoretic and expectorant. It increases the alkalinity of blood diminishes its tendency to coagulation and lessens its oxygen carrying power. Inhalation of vapour causes spasmodic closure of the glottis and suffocation. ${ }^{22}$ It is a venous medicine causing under-oxygenation causing of symptoms. Ammonium carb is also indicated in pneumonia when there is great debility, together with symptoms pointing to the formation of heart $\operatorname{clot}^{[27]}$.

Aspidosperma Quebracho: White Quebracho. N. O. Apocynaceae. Tincture and trituration of the bark. Trituration and solutions of the alkaloid Aspidospermine and its salts. Quebracho is a Brazilian fever remedy from which the alkaloid Aspidospermine has been isolated. Hale says "it produces in animals respiratory paralysis, slowed heart, and paralysis of extremities. It relieves dyspnoea in phthisis and pleurisy without fever. It is Indicated in Mitral incompetence and stenosis with severe nocturnal dyspnoea and Fatty heart with severe asthma. Severe Rales with pearls" of rounded gelatinous masses were expectorated. Aspidospermine in $3 x$ releives usually ${ }^{[22]}$.

Cardus MUR: Description. Natural Order, Compositae. Common Name, St. Mary's Thistle; Preparations: Mother tincture made from the ripe, whole seeds. Physiological Action-This agent acts upon the venous system, especially the portal vein. This remedy is indicated in diseases of the liver, portal system and the whole venous system. There is hardness and tenderness of the left lobe of the liver, pressure at this point causing oppressed breathing and cough ${ }^{[22]}$.

\section{Fluoric Acid}

Description. Hydrofluoric acid is a colorless fuming liquid, 
obtained by distilling fluor spar with Sulphuric acid. Dosage -Dilutions $3 \mathrm{X}$ and higher. At physiological level it is a strong escharotic, where its action extends deeply into the tissues causing a dry, painful slough. Its action upon the lower tissues causes caries of the bones, ulceration, varicose veins, derangement of the nails, hair, etc. ${ }^{[21]}$. Distended blood vessels, Varicosis.

Discussion: Coagulation is the main cause to prevent complications in covid19. In pulmonary complications Hypoxia leads to thrombus and in turn Thrombosis leads to hypoxia. The complication of coagulation leads to further damage further to multi organ failure. In coagulation resulting from Covid 19, D-Dimers are of diagnostic value which predicts the severity of Coagulation and assess the prognosis of diseases. Homoeopathic remedies though act well when selected on individualisation basis, in some acute emergency setups, specific remedies need to be given as thee need of the hour. Such remedies acting on Caogulation mechanism are Tissue remedies like Kalimur, Ferrum phos, and Natrum sulph. Remedies acting on clotting mechanism like Kalimur which also had equal affinity on fibrosis and alveolitis of lungs may help in relieving in the advanced stages of Covid disease. Remedy like Natrum sulph too help in relieving the Pneumonia especially in cases effecting the lower lobes. Ferrum phos though indicated in initial stages of this viral disease it can be preceded or followed by Kalimur as the case demands. The Ophidia group drugs like Lachesis, Bothrops, and Vipera too acts on the clotting system but Bothrops mostly indicated in after effects of injuries, while vipera in Deep vein thrombotic affections and Lachesis in threatening gangrene of the lungs. Though Lachesis is indicated in many Haemorrhagic conditions, rather clotting conditions, but in many advanced Repertories we find it under Paretic conditions of organs arising from Thrombotic conditions. Hence this remedy is to be thought of in coagulopathies. Drugs like Arnica does not had any invitro effects on Thrombolysis but invivo shows positive results due to interaction with some Endothelial factors. Remedies like Ammonium carb, Aspidosperma can be used to relieve the acute respiratory damage. Arsenic album already proved its beneficial effects in controlling the Covid-19 also needs to be researched further directly on Thrombolytic effects.

\section{Conclusion}

Covid-19 presenting as Acute viral pneumonia causes thrombotic complications leading to Respiratory failure and ultimately multi system dysfunction. The COVID-19associated coagulopathy (CAC) are distinct from those seen with bacterial Sepsis-Induced Coagulopathy (SIC) and disseminated intravascular coagulation (DIC). The CAC usually shows increased D-dimer and fibrinogen levels with minimal abnormalities in Prothrombin time and platelet count. The patients getting admitted for Covid-19 associated complications are to be monitored for D-Dimers and Coagulopathy profile before during and after admission especially for assessing the complications and Prediction of outcome result. Homoepathic remedies specified above like Kalimur, Natrum sulph, Arsenic alb, Ammonium carb can be thought of primarily as an adjuvant to conventional treatment for an early recovery.

\section{Acknowledgements}

My sincere gratitude for all the Co-faculties of NHRIMH for encouraging me in writing this publication.

\section{References}

1. Kit-San Yuen et al. SARS-2 and COVID -19, Cell Biosci. 2020; 10:40. Published online 2020, Mar. 16, doi: 10.1186/s13578-020-00404-4.

2. Richard C. Becker' J, COVID-19 update: Covid-19associated coagulopathy, Thrombolysis. 2020 May 15: 1-14. doi: 10.1007/s11239-020-02134-3.

3. Zhou P, Yang XL et al. A pneumonia outbreak associated with a new coronavirus of probable bat origin. 2020; 579(7798):270-273.- 03 February 2020

4. Tanaka.KA et al. Blood Coagulation: Hemostasis and Thrombin Regulation, Anesthesia and Analgesia, 01 May. 2009; 108(5):1433-1446.

5. Brenner B, Francis CW, Marder VJ. The role of soluble cross-linked fibrin in D-dimer immunoreactivity of plasmic digests". J Lab Clin Med. 1989; 113:682-8.

6. Pfitzner SA, Dempfle CE, Heene DL. Fibrin detected in plasma of patients with disseminated intravascular coagulation by fibrin-specific antibodies consists primarily of high molecular weight factor XIIIacrosslinked and plasmin-modified complexes partially containing fibrinopeptide. A. Thromb Haemost. 1997; 78:1069-78.

7. Wakai A, Gleeson D. Winter, Role of fibrin D-dimer testing in emergency medicine, Emerg.Med J: first published as 10.1136/emj.20.4.319 on 1 July 2003

8. Anoop J. Chauhan et al, COVID-19: a collision of complement, coagulation and inflammatory pathways, First published: 30 June, 2020.

9. Van Beusekom Mary. News Writer, Autopsies of COVID-19 patients reveal clotting concerns, Filed Under, COVID-19, | CIDRAP News, May 07, 2020

10. Marcel Levi et al, Coagulation abnormalities and thrombosis in patients with COVID-19, Published online- may, 11, 2020.

11. Jean M, Connors Jerrold, H Levy et al. Covid-19 and its implications for thrombosis and anticoagulation, Perspective June 4, 2020.

12. Tang N, Li D, Wang X, Sun Z. Abnormal coagulation parameters are associated with poor prognosis in patients with novel coronavirus pneumonia. J Thromb Haemost. 2020; 18(4).

13. Mr. Saskiamiddeldorp et al. Incidence of venous thromboembolism in hospitalized patients with COVID-19; 2020; 18(8):1995-2002. doi: 10.1111/jth.14888. Epub 2020 Jul 27.

14. Brandon $\mathrm{M}$ et al. Coagulopathy in Covid-19: Review and Recommendations"

15. Evangelos.Terpos et al. Hematological findings and complications of COVID-19. 13 April; https://doi.org/10.1002/ajh.25829; American Journal of Haematology. 2020; 95(7).

16. Paul Herscu ND, Dhanp MPH. Bothrops LanceolatusThe New England Journal of Homeopathy Fall/Winter. 1999; 8(2).

17. Coagulant and anticoagulant activities of Bothrops lanceolatus (Fer de lance) venom Toxicon- Volume 39, Issues 2-3, February-March, 2001, 371-37. 
18. Ana Paula Kawakami et al, Inflammatory Process Modulation by Homeopathic Arnica montana 6CH: The Role of Individual Variation, 2011. https://doi.org/ $10.1155 / 2011 / 917541$.

19. Jarne van stefan tander, KE Synclare. A dissertation on efficacy of Arnica 30 and 200 to thrombolyse a blood clot in an in-vitro sample - Johannesburg, 2005.

20. Schattauer GmbH - The Action of Vipera aspis Venom on Blood Clotting Factors and Platelets.

21. Robin Murphy. Homoeopathic Medical Repertory Second Revised Edition-Reprint 2004.B. Jain Publishers (P) Limited, New Delhi.

22. AL Blackwood. A Manual of Materia Medica, Therapeutics Pharmacology with Clinical Index Philadelphia, Boericke\&Tafel-1906

23. William H Burt. Characteristic Materia Medica Philadelphia - A.J. Tafel, 1869.

24. Dr. Hitesh Shah. Cellular Biochemistry and Homoeopathy - Reprint Edition -2004, Indian books and Periodical Publishers, Pg-228.243

25. Dr. Anil Khurana et al. Homoeopathic Perspectives in Covid-19 Coronavirus Infection"- Central Council for Research in Homoeopathy. Fact sheet- March, 2020.

26. William Boericke, Willis A Dewey. The Twelve Tissue Remedies of Schuessler Comprising the Theory Therapeutic Applications, Materia Medica and Complete Repertory Of these Remedies, Homoeopathically and Biochemically considered Philadelphia- Boericke Tafel, 1899.

27. Prof G Vithoulkas. International Academy of Classical Homoeopathy - Ammonium Carb.

28. Dr. W. H. Schuessler - The Biochemic Hand book Published by Plantscan Publications P.o Box 40, Bury St Edmunds, Suffolk IP3, 2SS. Copyright text @ , 1998. 